\title{
Amiodarone pulmonary toxicity: \\ a report on two cases
}

\author{
Ahmed Zedan MD, Osama Mukarram MD, Charles E. Burns MD, Yasir Ahmed MD
}

\begin{abstract}
Amiodarone is an iodine-containing antiarrhythmic drug commonly used for the treatment of ventricular and supraventricular arrhythmias. Amiodarone toxicity has several clinical presentations and affects multiple organs. One well-known serious side effect of amiodarone is pulmonary toxicity which can be acute, sub-acute, or chronic. Amiodarone pulmonary toxicity may present a diagnostic dilemma, particularly when clinical suspicion of pulmonary infection is high. Here we report two cases of amiodarone lung toxicity that were diagnosed in an early stage and improved after the discontinuation of amiodarone.
\end{abstract}

Key words: Amiodarone induced pneumonitis, amiodarone pulmonary toxicity, drug toxicity

\section{INTRODUCTION}

Amiodarone is commonly used for the treatment of supraventricular and ventricular arrhythmias. Due to high clinical efficacy, it is recommended by the American College of Cardiology / American Heart Association in atrial fibrillation, especially in patients with heart failure. ${ }^{1,2}$ Amiodarone tends to accumulate in certain organs, like skin, thyroid, eye, peripheral nerves, and lungs, and can cause bluish skin discoloration, photosensitivity, thyroid dysfunction, corneal deposits, peripheral neuropathy, bone marrow suppression, hepatitis, heart blocks, and pneumonitis. One well known serious side effect of amiodarone is pulmonary toxicity which can be acute, sub-acute, or chronic and can be irreversible and fatal. ${ }^{4,5}$ The diagnosis of amiodarone pulmonary toxicity is challenging

Corresponding author: Osama Mukarram MD Contact Information: Osama.mukarran@ttuhsc.edu DOI: 10.12746/swrccc2015.0310.134 and requires a high level of suspicion due to the nonspecific symptoms and image findings.

\section{CASE 1}

A 67-year-old man with a history of hypertension, coronary artery disease, ischemic cardiomyopathy with ejection fraction of $50 \%$, and atrial fibrillation controlled with amiodarone $200 \mathrm{mg}$ twice daily presented with progressive shortness of breath and dry cough for two weeks. He denied fever, chills, chest pain, orthopnea, and paroxysmal nocturnal dyspnea. He had been started on tapering high dose corticosteroids for intractable headache resulting from a subdural hematoma and amiodarone for atrial fibrillation six weeks prior to admission. Initial vital signs revealed temperature $98.7^{\circ} \mathrm{F}$, heart rate 85 beats/minute, respiratory rate 32 breaths/minute, blood pressure $135 / 80 \mathrm{mmHg}$, and oxygen saturation $80 \%$ on room air. Examination of the chest showed bilateral coarse crackles with no wheezes. No clinical signs of fluid 


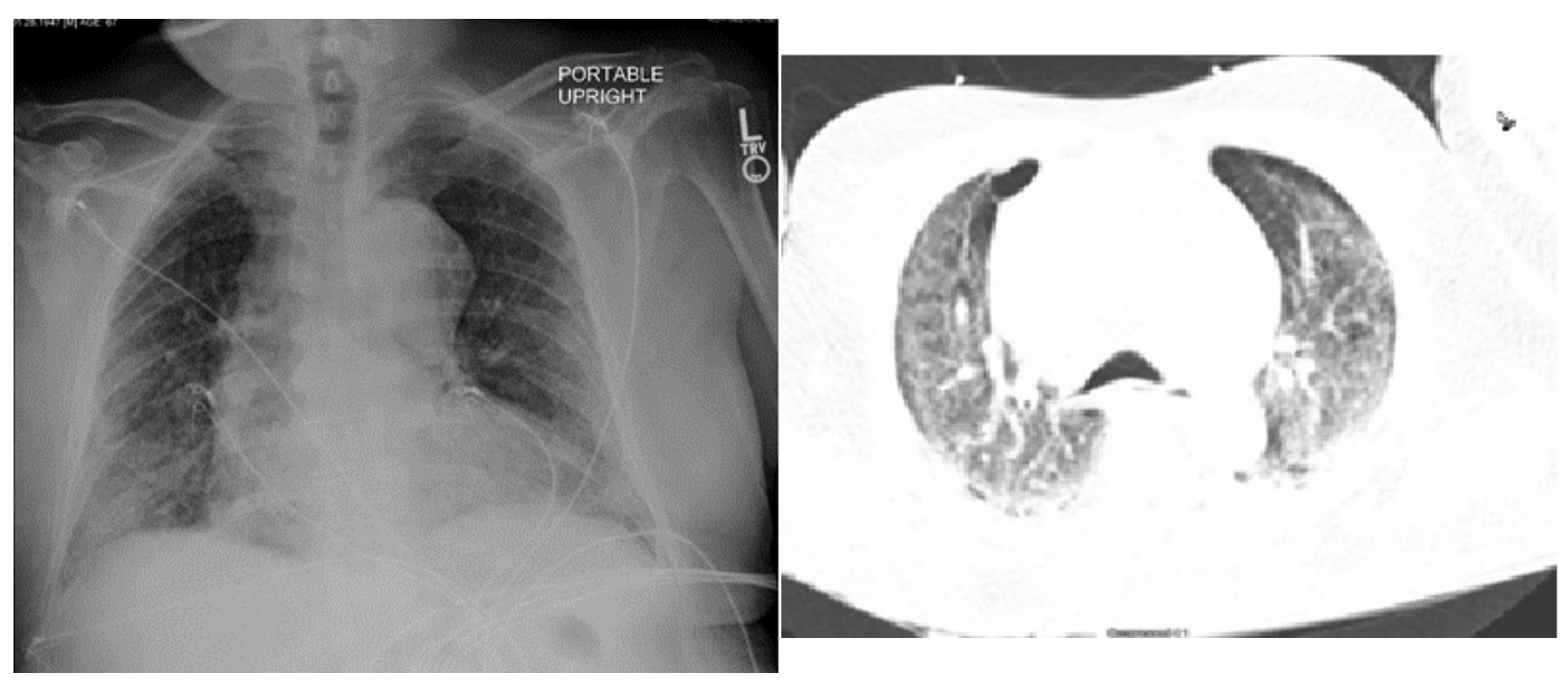

Figure1: 1a. Chest x-ray (AP view) shows bilateral interstitial pulmonary infiltrates. 1b. CT chest without contrast shows bilateral interstitial infiltrates and ground glass opacities.

overload were noted on the physical examination, and the remainder of his examination was unremarkable. A chest $x$-ray showed diffuse bilateral interstitial infiltrates, and computed tomography (CT) chest showed ground glass opacities with suspected pneumonia (Figure 1a, 1b). Initial laboratory work yielded normal blood cell counts, normal electrolytes, and cardiac enzymes. Arterial blood gas showed $\mathrm{pH} 7.51, \mathrm{PaCO}_{2}$ $35.2 \mathrm{mmHg}, \mathrm{PaO}_{2} 59 \mathrm{mmHg}, \mathrm{HCO}_{3} 28.2 \mathrm{mmol} / \mathrm{L}$ on $100 \% \mathrm{FiO}_{2}$. Based on his recent history of high dose corticosteroids and radiographic results, empiric clindamycin and primaquine treatment for suspected Pneumocystis jiroveci pneumonia (PJP) along with vancomycin and levofloxacin was begun. Amiodarone was discontinued. His hypoxia worsened, and he required intubation and mechanical ventilation within 48 hours. All tests for infection, including blood cultures, fungal serologies, sputum and bronchoalveolar lavage (BAL) bacterial, fungal, acid fast bacilli cultures, PJP PCR, and HIV screen, remained negative. Transthoracic echocardiogram showed an ejection fraction of $40-45 \%$, mild left ventricular hypertrophy, and grade II diastolic dysfunction. Due to persistent pulmonary infiltrates with no obvious etiology, the patient eventually underwent a lung biopsy that showed patchy interstitial pneumonitis with foci of interstitial fibrosis, reactive hyperplasia of pulmonary macrophages, many type 2 pneumocytes, and widespread foamy cytoplasmic changes consistent with amiodarone toxicity (Figure $2 a, 2 b$ ). The patient was started on $60 \mathrm{mg}$ daily prednisone, and his respiratory condition started to improve. He was able to be weaned from the ventilator, extubated, and went home on 2 liters per minute oxygen.

\section{CASE 2}

A 79-year-old Caucasian man with dementia, hypertension, paroxysmal atrial fibrillation, and chronic obstructive lung diseases requiring 3 liters per minute of continuous oxygen at home was admitted for chronic constipation and abdominal pain. On hospital day (HD) three he developed shortness of breath, cough, and a fever of $102^{\circ} \mathrm{F}$. His vital signs included blood pressure $130 / 85 \mathrm{mmHg}$, heart rate 98 beats/minute, respiratory rate 18 breaths/minute, and oxygen saturation $95 \%$ on $40 \% \mathrm{FiO}_{2}$ via venturi mask. His chest examination showed bilateral coarse crackles and wheezes. The remainder of the physical 


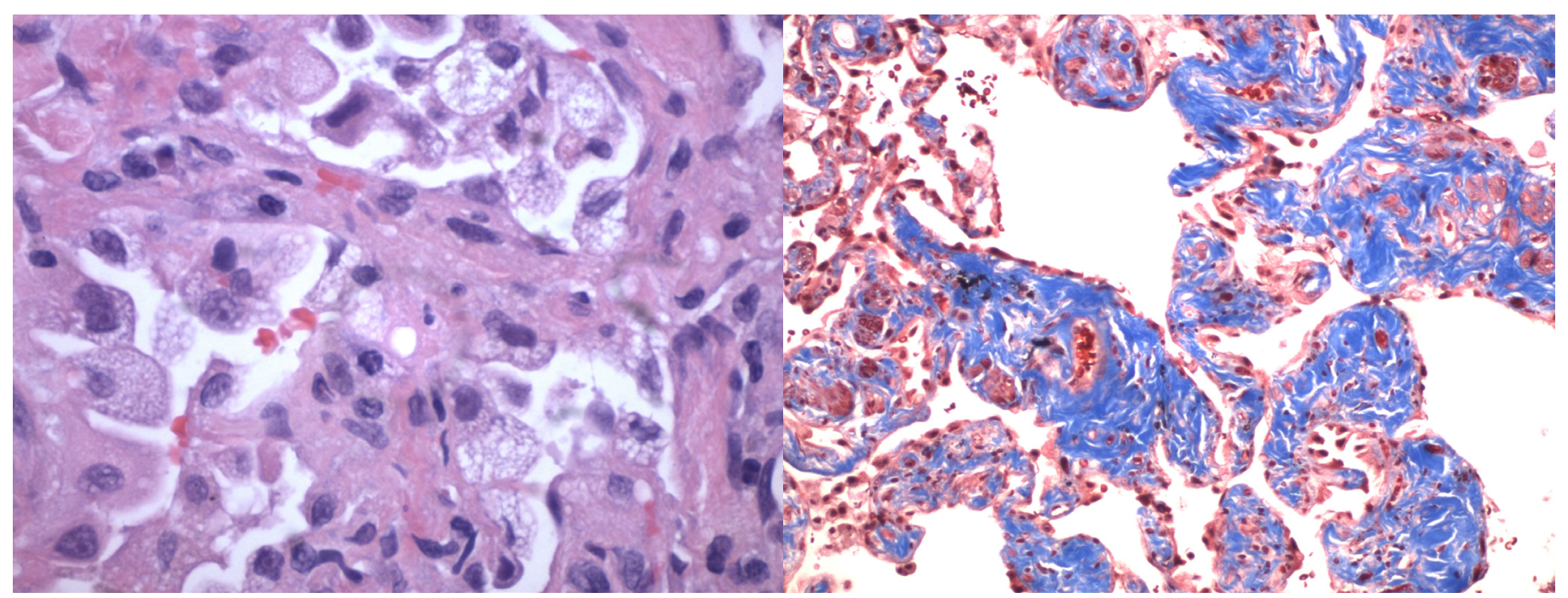

Figure2a: Lung wedge biopsy shows alveolar septal thickening, interstitial chronic inflammation, and fibrosis. The intra-alveolar macrophages and some Type II pneumocytes display the distinctive fine cytoplasmic vacuolization characteristic of amiodarone pulmonary toxicity (400X, H\&E stain).
Figure2b: Lung wedge biopsy, same specimen as 2a. $100 \mathrm{X}$, Trichrome stain. The alveolar septae demonstrate varying degrees of fibrosis, from nearly normal appearance in upper left corner to prominent thickening with mature collagen which stains blue. examination was unremarkable. Laboratory work up showed white blood cell count (WBC) $29.2 \times 10^{3} / \mu \mathrm{L}$ (polymorphs $73 \%$, lymphocytes $22 \%$ ), hemoglobin 11 $\mathrm{gm} / \mathrm{dL}$, platelets $420 \times 10^{3} / \mu \mathrm{L}$, lactic acid of $0.6 \mathrm{mmol} / \mathrm{l}$ (normal 0.5-2.2), blood urea nitrogen $41 \mathrm{mg} / \mathrm{dL}$, creatinine $1.5 \mathrm{mg} / \mathrm{dL}$, and normal liver enzymes. Chest x-ray showed left lower lobe and right lower lobe lung infiltrates that were not present at admission. Empiric vancomycin and piperacillin-tazobactam therapy was begun. Blood and sputum cultures remained negative. On HD 12, his pneumonia had improved, but he developed atrial fibrillation with a rapid ventricular rate of 130 beats/minute and was started on an amiodarone drip that was subsequently switched to 200 mg oral daily. On HD 22, he developed worsening of respiratory symptoms and hypoxemia with increased oxygen requirement up to $50 \% \mathrm{FiO}_{2}$ via vapotherm. Vital signs included temperature $98.7^{\circ} \mathrm{F}$, blood pressure 110/65 $\mathrm{mmHg}$, and pulse rate 80 beats/minute. Examination of the lungs revealed bilateral crackles with mild wheezes. Laboratory work revealed a normal WBC count without bands and normal serum electrolytes. Arterial blood gas revealed $\mathrm{PH} 7.266$, $\mathrm{PaCO}_{2} 73.2 \mathrm{mmHg}, \mathrm{PaO}_{2} 67 \mathrm{mmHg}$, and $\mathrm{HCO}_{3} 33$ $\mathrm{mmol} / \mathrm{L}$. Chest $\mathrm{x}$-ray showed haziness, predominantly over the left lung, with mild, diffuse lung infiltrates (Figure-3a). A CT of the chest without contrast showed reticulonodular interstitial infiltrates scattered through both lungs, most prominent posteriorly and at the periphery of the lungs (Figure-3b). Echocardiogram showed an ejection fraction of $50 \%$ and a right ventricular systolic pressure of 50 to $60 \mathrm{mmHg}$. At this point the patient's family mentioned a possible adverse reaction to amiodarone in the past the patient did not report at admission. Apparently three years ago the patient received amiodarone for atrial fibrillation and developed a dry cough with hypoxia. Amiodarone was discontinued, and his clinical symptoms resolved promptly. No tissue biopsy or chest imaging was done at that time. Based on this past strong amiodarone pulmonary toxicity history and current pulmonary finding with rechallenge of amiodarone, the clinical diagnosis of amiodarone pulmonary toxicity was made. Amiodarone was discontinued, and 


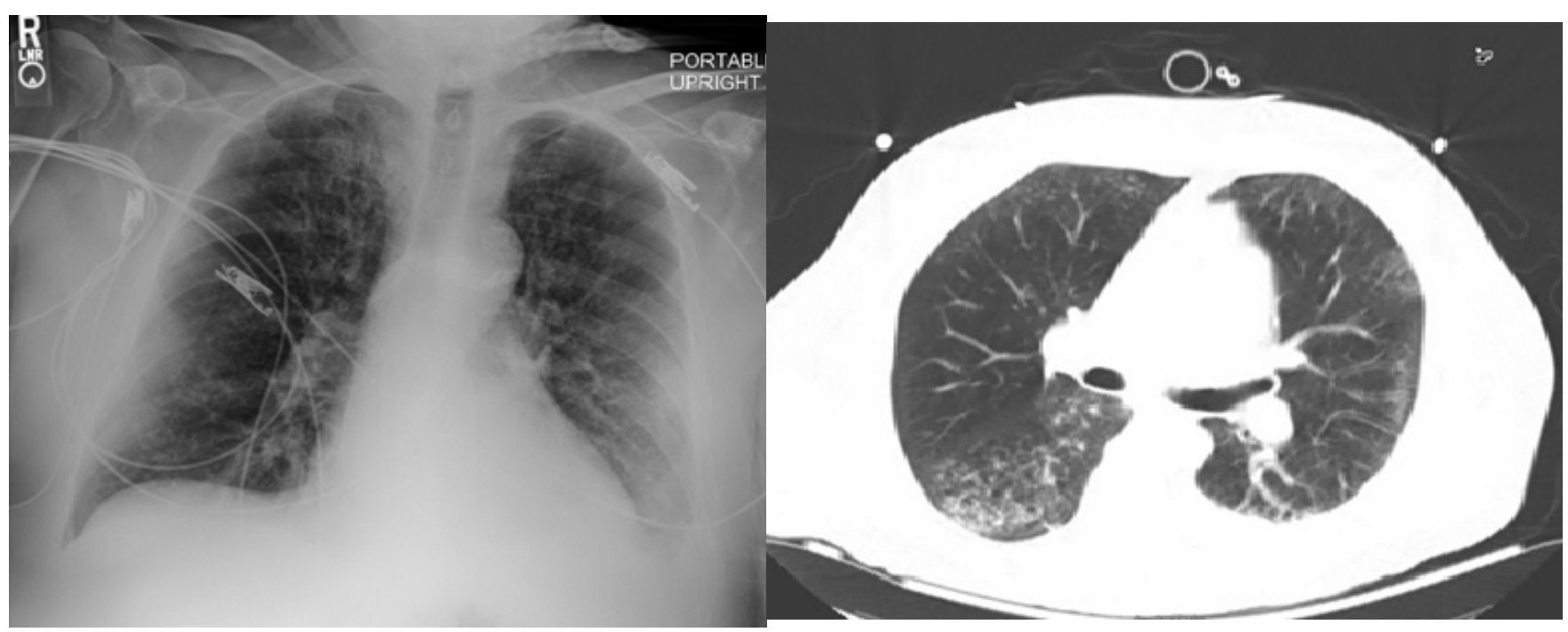

Figure3: 3a. Chest x-ray (AP portable) shows bilateral interstitial pulmonary infiltrates with blunting of the left costophrenic angle, possibly due to a small pleural effusion. 3b. CT chest without contrast shows patchy reticulonodular interstitial infiltrates in both lungs.

the patient's respiratory symptoms improved. He was started on a short tapering course of corticosteroids, and his oxygen requirements improved to his baseline of 3 liters per minute via nasal cannula.

\section{Discussion}

Amiodarone is an iodinated benzofuran derivative containing Vaughan-Williams class III antiarrhythmic agent. Initially it was used as anti-anginal agent due to its vasodilator properties and later in 1970 s as an antiarrhythmic agent for both ventricular and supraventricular tachycardia with significant efficacy. Amiodarone controls arrhythmia by inhibiting the influx of sodium and calcium into the cardiac tissue resulting in suppression of excitability and conductivity of cardiac tissues, especially in myocytes. It also inhibits voltage and ligand-gated potassium channel currents, and this prolongs the action potential in cardiac tissues. Due to high lipid solubility and volume of distribution, it has a prolonged half-life. Amiodarone easily accumulates in adipose tissue and highly vascular organs, like the liver, lungs, and spleen. ${ }^{7}$ It is metabolized in the liver by cytochrome P-450 enzyme with subsequent excretion via the biliary sys- tem; it is not dialyzable in overdose intoxication. ${ }^{7,8}$

Amiodarone pulmonary toxicity is a common and serious side effect of amiodarone, but the exact mechanism for this toxicity is unclear. ${ }^{9}$ Some authors have postulated that direct cytotoxic lung injury or an indirect hypersensitivity reaction are the main mechanisms. ${ }^{10}$ The risk for amiodarone pulmonary toxicity is associated with a high cumulative dose of amiodarone rather than plasma concentration or daily dose. ${ }^{4}$ Patients taking $400 \mathrm{mg}$ daily for two months or 200 $\mathrm{mg}$ for two years are at high risk of amiodarone pulmonary toxicity with reported incidence of $5-15 \%{ }^{11}$ Other risk factors for amiodarone pulmonary toxicity include male gender, advanced age, preexisting lung disease and high oxygen requirements, thoracic surgery, and pulmonary angiography.

The differential diagnosis of amiodarone pulmonary toxicity includes bronchiolitis obliterans, chronic eosinophilic pneumonia, bronchiolitis obliterans organizing pneumonia, and interstitial pneumonitis. In patients with chronic underlying cardiopulmonary diseases it is sometimes challenging to diagnose amiodarone pulmonary toxicity due to similar clinical 
and radiological presentations. A temporal relationship of amiodarone intake for months or years can be a clue for this diagnosis. ${ }^{14}$

There is no specific test to diagnose amiodarone pulmonary toxicity. The diagnosis is based on clinical presentation, radiographic imaging, and histopathological findings and is mainly a diagnosis of exclusion. ${ }^{11,15}$ The clinical presentation of amiodarone pulmonary toxicity is very nonspecific. Common symptoms are shortness of breath, dry cough, fever, respiratory distress, and fatigue; sometimes it can mimic acute respiratory distress syndrome. ${ }^{16}$ The initial radiological work up is the chest $x$-ray which can shows patchy or diffuse infiltrates that are commonly bilateral and often have a ground glass appearance. High resolution CT scans show bilateral interstitial, alveolar, or mixed interstitial and alveolar infiltrates and ground glass opacities. ${ }^{17}$ Pleural thickening, effusions, and upper lung lobe nodules secondary to accumulation of iodine in type II pneumocytes have also been reported in the literature. ${ }^{18}$ Gallium scanning with increase uptake of gallium can predict an inflammatory process, but this is a nonspecific result. ${ }^{19}$ Pulmonary function tests will show a restrictive pattern with a decreased diffusion capacity. BAL may help rule out other causes of interstitial pneumonitis. The presence of foam cells is often present, but this is a nonspecific finding. Polymorphonuclear leukocytes and T suppressor CD8+ cells are commonly seen in BAL fluid.

Lung biopsy should probably be avoided due to concern for possible clinical worsening of respiratory symptoms in patients with amiodarone following thoracic surgical procedure except when the diagnosis is unclear. ${ }^{11}$ Lung biopsy usually shows alveolar septal widening with inflammatory infiltrates, type II pneumocyte hyperplasia, interstitial fibrosis, and diffuse interstitial pneumonitis, as in our first case. ${ }^{21} A$ peculiar type of foamy macrophages that tend to accumulate in alveolar spaces is a characteristic finding although that correlates primarily with exposure to amiodarone. It is not diagnostic of amiodarone toxicity per se since it has been reported in patients undergoing chronic amiodarone therapy who do not have evidence of amiodarone pulmonary toxicity. ${ }^{18,20}$ The absence of these cells makes the diagnosis of amiodarone toxicity less likely.

The main treatment of amiodarone pulmonary toxicity is discontinuation of the drug. The disease may progress initially due to the prolonged half-life and accumulation of the drug in the adipose tissue. Corticosteroids (prednisone 40-60 mg daily) can be given for a prolonged period with slow tapering over months. About $75 \%$ of cases with amiodarone pulmonary toxicity have a favorable outcome after discontinuation of the drug with or without the addition of corticosteroids. ${ }^{22}$ Recurrent amiodarone pulmonary toxicity on rechallenge with amiodarone can confirm the clinical diagnosis but generally is not recommended due to the potential serious outcome. Magro, et al. reported recurrent amiodarone pulmonary toxicity in four out of six patients with low dose amiodarone rechallenge. Two of these four patients developed signs of pulmonary toxicity despite use of concomitant steroid therapy. The time delay between recurrence of pulmonary symptoms and re-initiation of amiodarone was not reported. ${ }^{23}$ Our first patient had acute amiodarone pulmonary toxicity and was diagnosed in the early stage. The second patient had a prior history of amiodarone induced pneumonitis, and his respiratory symptoms and radiological findings worsened with unintentional re-challenge of amiodarone. This resolved immediately upon discontinuation of amiodarone and a short course of corticosteroids.

In summary, amiodarone pulmonary toxicity is rare, especially with early onset, and may present a diagnostic dilemma. Close monitoring and vigilance are required for early recognition of amiodarone pulmonary toxicity. Most patients respond favorably, if the diagnosis is made in a timely manner, to discontinuation of amiodarone with or without the use of corticosteroids. 
Author Affiliation: Ahmed Zedan and Osama Mukarram are residents in Internal Medicine at Texas Tech University Health Sciences Center in Odessa, TX. Charles E. Burns is a pathologist at Medical Center Hospital in Odessa, TX. Yasir Ahmed is an infectious disease specialist in the Department of Internal Medicine at TTUHSC in Odessa, TX.

Received: 02/25/2015

Accepted: 03/20/2015

Reviewers: Rishi Raj MD

Published electronically: 04/15/2015

Conflict of Interest Disclosures: none

\section{REFERENCES}

1. January CT, Wann L, Alpert JS, Calkins H, Cigarroa JE, Cleveland JC et al. 2014 AHA/ACC/HRS Guideline for the Management of Patients With Atrial Fibrillation: Executive Summary. J Am Coll Cardiol 2014; 64(21):2246-2280.

2. Kodama I, Kamiya K, Toyama J. Amiodarone: ionic and cellular mechanisms of action of the most promising class III agent. Am J Cardiol 1999 Nov 4; 84(9A):20R-28R.

3. Mason JW. Amiodarone. N Engl J Med 1987; 316:455.

4. Wolkove N, Baltzan M. Amiodarone pulmonary toxicity. Can Respir J 2009 Feb; 16(2): 43-48.

5. Goldschlager N, Epstein AE, Naccarelli GV, Olshansky B, Singh B, Collard HR, Murphy E. A practical guide for clinicians who treat patients with amiodarone: 2007. Heart Rhythm 2007 Sep; 4(9):1250-9.

6. Punnam S, Goyal S, Kotaru V, Pachika A, Abela G, Thakur R.: Amiodarone - a 'broad spectrum' antiarrhythmic drug. Cardiovasc Hematol Disord Drug Targets 2010; 10: pp. 73-812.

7. Vassallo P, Trohman RG. Prescribing amiodarone. JAMA 2007; 298:1312-22.

8. Zimetbaum P. Amiodarone for atrial fibrillation. $N$ Engl J Med 2007; 356:935-41.10: 4pp. 73-81

9. Leeder RG, Rafeiro E, Brien JF, Mandin CC, Massey TE. Evaluation of reactive oxygen species involvement in amiodarone pulmonary toxicity in vivo and in vitro. $J$ Biochem Toxicol 1996; 11 (3): 147-160.

10. Martin WJ 2nd, Rosenow EC 3rd. Amiodarone pulmonary toxicity. Recognition and pathogenesis (Part I). Chest. 1988; 93 (5): 1067-1075.
11. Jessurum GA, Crijns HJG. Amiodarone pulmonary toxicity. BMJ 1997; 314:619-20.

12. Ernawati DK, Stafford L, Hughes JD. Amiodarone-induced pulmonary toxicity. Br J Clin Pharmacol 2008; 66 (1): 82-87.

13. Handschin AE, Lardinois D, Schneiter D, Bloch K, Weder W. Acute amiodarone-induced pulmonary toxicity following lung resection. Respir Int Rev Thorac Dis 2003; 70 (3): 310-312.

14. Kudenchuk PJ, Pierson DJ, Greene HL, Graham EL, Sears GK, Trobaugh GB. Chest 1984; 86(4):541-548.

15. Kanji Z, Sunderji R, Gin K. Amiodarone-induced pulmonary toxicity. Pharmacotherapy 1999; 19:1463-1466.

16. Ott MC, Khoor A, Leventhal JP, Paterick TE, Burger CD. Pulmonary toxicity in patients receiving low-dose amiodarone. Chest 2006; 123:646-51.

17. Kuhlman JE, Teigen C, Ren H, Hurban RH, Hutchins GM, Fishman EK. Amiodarone pulmonary toxicity: CT findings in symptomatic patients. Radiology 1990; 177:121-125.

18. Dharmarajan TS, Shah AB, Dharmarajan L. Amiodaroneinduced pulmonary toxicity: Potentially fatal, recognize early during life! J Am Geriatr Soc 2008; 56:1363-1365.

19. Fabiani I, Tacconi D, Grotti S, Brandini R, Salvadori C, Caremani M, Bolognese L. Amiodarone-induced pulmonary toxicity mimicking acute pulmonary edema. J Cardiovasc Med (Hagerstown) 2011; 12:361-365.

20. Camus P, Martin WJ II, Rosenow EC III. Amiodarone pulmonary toxicity. Clin Chest Med 2004; 25:65-75.

21. Marchlinski FE, Gansler, Waxman HL, Josephson ME. Amiodarone pulmonary toxicity. Ann Int Med 1982; 97:839-45.

22. Yamada Y, Shiga T, Matsuda N, Hagiwara N, Kansanuki $\mathrm{H}$. Incidence and predictors of pulmonary toxicity in Japanese patients receiving low-dose amiodarone. Circ J 2007; 71:1610-6.

23. Magro SA, Lawrence EC, Wheeler SH, Krafchek J, Lin HT, Wyndham CR. Amiodarone pulmonary toxicity: prospective evaluation of serial pulmonary function tests. $\mathrm{J} \mathrm{Am}$ Coll Cardiol 1988 Sep; 12(3):781-8. 